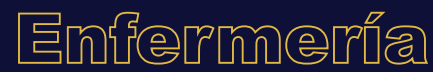

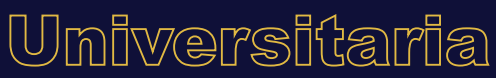

\section{Análisis de la distribución del tiempo en materia de cuidado crítico por profesionales de enfermería mediante TISS-28}

\section{Analysis of the distribution of time in critical care by nursing professionals using TISS-28}

\section{Análise da distribuição do tempo em cuidados intensivos por profissionais de enfermagem usando o TISS-28}

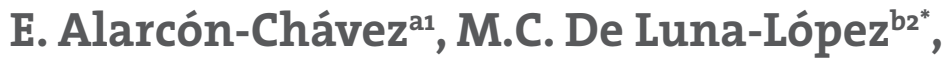 L. Flores-Padilla ${ }^{\mathrm{c}}$, M. Jaramillo-Beltrán ${ }^{\mathrm{d} 4}$, L.J. Gómez-Jurado ${ }^{\mathrm{e}}$, M.I. Brito-Cerino ${ }^{\mathrm{f5}}$}

\section{ORCID}

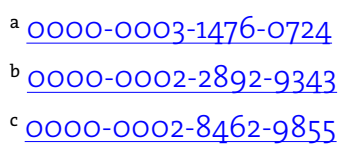

\author{
d \\ e $0000-0003-4351-2332$ \\ f $0000-0002-0935-9031$
}

${ }^{1}$ Instituto Mexicano del Seguro Social, Hospital General de Zona No. 6, Departamento de Enfermería, Ciudad Juárez, Chihuahua, México

${ }^{2}$ Instituto Mexicano del Seguro Social, Hospital General de Zona No. 6, Coordinación de Programas de Enfermería, Ciudad Juárez, Chihuahua, México

${ }^{3}$ Universidad Autónoma de Ciudad Juárez, Instituto de Ciencias Biomédicas, Ciudad Juárez, Chihuahua, México

4 Instituto Mexicano del Seguro Social, Hospital General de Zona No. 35, Departamento de Enfermería, Ciudad Juárez, Chihuahua, México

5 Instituto Mexicano del Seguro Social, Unidad de Medicina Familiar No. 34, Departamento de Enfermería, Ciudad Juárez, Chihuahua, México

Recibido: 14 octubre 2019

Aceptado: 25 noviembre 2019

*Autora de correspondencia. Correo electrónico: conyxo6@gmail.com 


\section{RESUMEN}

Introducción: Hoy en día, las intervenciones terapéuticas son más especializadas y complejas. Se ha identificado que el Therapeutic Intervention Scoring System-28 (TISS-28) es un instrumento adecuado para planear las actividades de enfermería.

Objetivo: Determinar mediante la aplicación del instrumento TISS-28 el grado de complejidad de las intervenciones realizadas al brindar cuidados de enfermería a pacientes en estado crítico.

Métodos: Estudio descriptivo, 270 pacientes (adultos, pediátricos y neonatales) en estado crítico; se recabó información sociodemográfica tanto del personal de enfermería como de los pacientes a través de un instrumento, se aplicó el TISS-28 para identificar el grado de complejidad de las intervenciones que se le brindaron al paciente. El análisis estadístico fue descriptivo para variables sociodemográficas y laborales, para las acciones realizadas por enfermería mediante niveles de grados de complejidad se hizo uso del análisis bivariado y la regresión logística.

Resultados: Las intervenciones brindadas por el personal de enfermería en el servicio de medicina interna en el Grado II es de 9.8 veces más que en el resto de los pacientes, grado III aumenta 68 veces cuando el paciente tiene una sobre estancia hospitalaria.

Discusión: Los pacientes que se encuentran en los servicios de cuidados intensivos o quirúrgicos requieren mayores cuidados.

Conclusión: El TISS-28 permite predecir el estado del paciente crítico y su evolución. Determina el tiempo de atención requerido según la gravedad de este, además facilita la asignación idónea de enfermera-paciente.

Palabras clave: Planificación de atención al paciente; gravedad del paciente; enfermería de cuidados críticos; carga de trabajo; unidades de cuidados intensivos; México.

\section{ABSTRACT}

Introduction: Currently, therapeutic interventions have become more complex and specialized, but the Therapeutic Intervention Scoring System-28 (TISS-28) has been identified as an adequate tool in the planning of the corresponding nursing activities.

Objective: To estimate through the Therapeutic Intervention Scoring System-28 (TISS-28) the degree of complexity of the necessary nursing interventions to provide care to patients in critical status.

Methods: This is a descriptive study on 270 adult, pediatric, and neonatal patients in critical status. The TISS-28, and nursing and patient sociodemographic instruments were used. A descriptive statistical analysis using the sociodemographic and work-related variables was carried out. Bi-variate analysis and logistic regression were calculated in order to analyze the nursing performance by levels of complexity.

Results: The interventions provided by the nursing staff in the internal medicine service in Grade II is 9.8 times more than in the rest of the patients, grade III increases by 68 times when the patient has an excess hospital stay.

Discussion: Patients in the intensive care or surgical services require more care.

Conclusion: The TISS-28 offers an estimate on the evolution and attention time required by patients in critical status as well as the corresponding ideal nurse-patient ratios.

Keywords: Patient care planning; patient acuity; critical care nursing; workload; intensive care units; Mexico. 


\section{RESUMO}

Introdução: Atualmente as intervenções terapêuticas são mais especializadas e complexas, identificou-se que o Therapeutic Intervention Scoring System-28 (TISS-28) é um instrumento adequado para planejar as atividades de enfermagem.

Objetivo: Determinar mediante a aplicação do instrumento TISS-28, o grau de complexidade das intervenções realizadas na assistência de cuidados de enfermagem a pacientes em estado crítico.

Métodos: Estudo descritivo, 270 pacientes (adultos, pediátricos e neonatais) em estado crítico; foi coletada informação sociodemográfica tanto do pessoal de enfermagem quanto dos pacientes através de um instrumento, aplicou-se o TISS-28 para identificar o grau de complexidade das intervenções que foram prestadas ao paciente. A análise estatística foi descritiva para variáveis sociodemográficas e laborais; a análise bivariada e a regressão logística foi utilizada para as ações realizadas por enfermagem mediante níveis de graus de complexidade.

Resultados: As intervenções realizadas pela equipe de enfermagem no serviço de clínica médica do Grau II são 9,8 vezes mais do que no restante dos pacientes, o grau III aumenta em 68 vezes quando o paciente tem um tempo de internação excessivo.

Discussão: Os pacientes que se encontram nos serviços de cuidados intensivos ou cirúrgicos requerem maiores cuidados.

Conclusão: O TISS-28 permite prever do estado do paciente crítico e sua evolução. Determina o tempo de atenção requerido conforme a gravidade deste, aliás facilita a atribuição idónea de enfermeira-paciente.

Palavras chave: Planejamento de assistência ao paciente; gravidade do paciente; enfermagem de cuidados críticos; carga de trabalho; unidades de terapia intensiva; México.

\section{INTRODUCCIÓN}

La atención del paciente crítico constituye un verdadero reto para el personal de salud al tener que brindar los cuidados especializados que son necesarios para los pacientes. Durante esta atención la vida está comprometida, por lo que se requiere un control riguroso como una estricta y continua monitorización. Debido a esto, la Unidad de Cuidados Intensivos (UCI) ocupa un lugar destacado en la atención del paciente en estado crítico‥ El índice de mortalidad es un indicador para evaluar el desempeño profesional en estas unidades, también se considera el principal marcador asistencial en la práctica médica. La mortalidad en estos servicios varía entre 10\% y $74 \%$. Se conoce que la mortalidad de la UCI está asociada con la gravedad del paciente, el nivel de intervención terapéutica y la necesidad de ventilación mecánica. En los pacientes críticos con puntajes altos de Acure Physiology \& Chronic Health Evalutation II (APACHE II) y Therapeutic Intervention Scoring System 28 (TISS-28) se presenta mayor riesgo de fallecer, particularmente de forma temprana².

Es importante resaltar que las intervenciones para el cuidado ocupan un lugar central y fundamental enla disciplina que contribuye en la recuperación del paciente ${ }^{3.4}$.El nivel de conocimiento del personal de enfermería y los años de experiencia en la UCI tienen una relación directa con los resultados en la aplicación del cuidado enfermero y la seguridad de los pacientes5. Hoy en día, las intervenciones terapéuticas son más especializadas en virtud de la gravedad de la enfermedad y 
también se ha identificado que el TISS-28 es una herramienta adecuada para planear las actividades de enfermería en los pacientes pediátricos, particularmente en los que son post-operados. Además, la literatura internacional menciona que la complejidad de la atención se ha asociado con la necesidad de un mayor número de profesionales de la salud ${ }^{6,7}$. El TISS-28 permite identificar la intensidad de la intervención terapéutica por el profesional de enfermería, asimismo determina el nivel de gravedad que presentó el paciente crítico, y es un predictor valioso para cuantificar la carga de trabajo del profesional de enfermería ${ }^{8}$. Este contexto define la importancia de medir las intervenciones del cuidado de enfermería, en donde existen múltiples instrumentos que auxilian a clasificar el nivel de gravedad de los pacientes y permiten identificar el tiempo requerido de la atención de enfermería. Uno de dichos instrumentos es el Sistema de Puntuación de Intervenciones Terapéuticas TISS-28, el cual ha sido utilizado para valorar las intervenciones asistenciales ejercidas por el profesional de enfermería, tanto en pacientes pediátricos como en adultos9.

Los primeros esfuerzos en la creación de estas herramientas corresponden a Cullen y Civetta, quienes por medio de la observación, desarrollaron en 1974 un instrumento para medir las intervenciones asistenciales debido al incremento de tiempo en la atención del cuidado enfermero durante la hospitalización que presentaban los pacientes críticos. Actualmente, el TISS-28 es una herramienta indispensable para medir los tiempos de trabajo en enfermería ${ }^{10}$. Algunos autores han planteado la necesidad de la utilización de este instrumento para la planeación de la atención en los pacientes y la optimización de los recursos humanos disponibles, pues se enfatiza que cuando enfermería brinda las intervenciones adecuadas, tendrá como consecuencia la recuperación de los pacientes ${ }^{11-13}$. El objetivo del estudio fue determinar mediante la aplicación de la herramienta TISS-28 el grado de complejidad de las intervenciones realizadas para brindar el cuidado de enfermería a pacientes en estado crítico.

\section{MÉTODOS}

Se realizó un estudio descriptivo, transversal y observacional en un hospital público de segundo nivel de atención médica. La población de estudio fueron usuarios de los servicios de medicina interna y cirugía. Se obtuvo una muestra probabilística con $95 \%$ de confianza y un margen de error del $5 \%$ a partir de los egresos hospitalarios de 2018. El muestreo fue al azar mediante el censo diario, del cual se eligió uno de cada tres pacientes. La población del estudio fue conformada por 270 pacientes adultos, pediátricos y neonatales en estado crítico, atendidos en los diferentes departamentos del hospital en Ciudad Juárez, Chihuahua, durante el primer semestre del año 2019. Se incluyó a pacientes con diagnóstico de estado crítico de salud. Se utilizó el instrumento Score TISS-28 (Therapeutic Intervention Scoring System 28), elaborado y validado por Cullen.

El instrumento se clasifica por sistemas: (actividades básicas 1-5 puntos, soporte ventilatorio 1-5 puntos, soporte cardiovascular 3-8 puntos, soporte renal 2-3 puntos, soporte neurológico 4 puntos, soporte metabólico 2-4 puntos e intervenciones especificas 3-5 puntos). El método y la interpretación de las intervenciones terapéuticas son a través de la suma de estos puntos de respuesta diagnóstica como terapéutica implementada, con una calificación desde 0 hasta 8 puntos, con un total de 28 medidas, cada punto corresponde a 10.6 minutos de atención profesional por enfermería dedicado al paciente crítico. Se obtuvo un alfa de Cronbach $=0.90$, lo que se considera muy aceptable ${ }^{10}$.De igual forma, con la puntuación de los grados del TISS-28 se determinó la complejidad de la atención del paciente: pacientes con menos de 10 puntos de observación Grado I, de 10 a 19 puntos se consideró vigilancia activa o Grado II, 20 a 30 puntos, vigilancia intensiva o Grado III y puntuación igual 
o mayor de 40 puntos terapéutica intensiva o Grado IV. La aplicación del instrumento se realizó durante una jornada laboral de ocho horas. También se incluyó una sección para identificar variables sociodemográficas de los pacientes (edad, género, servicio de atención médica y días de estancia hospitalaria), así como del personal de enfermería (género, edad, estado civil, antigüedad, turno y categoría laboral).

Para la recolección de datos se capacitó a dos enfermeras para que acudieran a los diferentes servicios hospitalarios de los turnos matutino, vespertino y nocturno, los siete días de la semana durante los meses de abril-agosto de 2019. El estudio se apegó a la Ley General de Salud en Materia de Investigación Para la Salud, se sometió a dictamen del Comité de Investigación y Ética del hospital, se obtuvo la autorización con registro 2019-801-004, además se solicitó permiso a las autoridades del hospital, al igual que el consentimiento informado a los pacientes y enfermeras participantes.

Se incluyó a pacientes en estado crítico de los diferentes servicios hospitalarios con una permanencia por más de 24 horas. La distribución del personal de enfermería era en equipo: un auxiliar de enfermería y una enfermera general por turno para atender entre ocho a diez pacientes. La información fue recabada diariamente a la misma hora, el responsable de la recolección de información era un profesional especialista en Cuidados Intensivos, lo que brindó certeza en el juicio clínico y aplicación del sistema TISS-28, midiendo las actividades, tiempos y la clasificación del nivel de gravedad de los pacientes. A los pacientes se les aplicó un cuestionario auto administrado para identificar variables sociodemográficas, de la atención médica y de enfermería. Se excluyó al personal de enfermería que no se encontraba vinculado directamente con la atención del paciente, jefes de servicio, pasantes y estudiantes de enfermería, se eliminó la encuesta con una cifra menor al 95\% de su registro, al profesional que laboró tiempo incompleto al utilizar pase de salida, personal que no deseara participar a la hora de la encuesta y pacientes que fallecieron con menos de 24 horas de estancia.

Una vez recolectados los datos y registrados, se procesaron en el paquete estadístico para ciencias sociales (SPSS) versión 22. Se realizaron pruebas de estadística descriptiva para las variables sociodemográficas y laborales.

\section{RESULTADOS}

Se incluyeron a 270 pacientes críticos, el género predominante fue el femenino con $54.1 \%$, los adultos de 36-64 años corresponden el 50.4\%, seguido del adulto mayor con el 30.7\%. Dos terceras partes de los participantes eran atendidos en el servicio de medicina interna. La duración de la estancia hospitalaria más frecuente fue de 1-7 días (76.3\%). Estos datos se encuentran descritos en la Tabla 1.

Con relación a las variables sociodemográficas del profesional de enfermería, el 73.7\% representa al género femenino, poco más de dos terceras partes tienen edad entre 18 a 39 años. Respecto a la escolaridad, la mitad manifiestan una educación media superior y el $44.1 \%$ curso la licenciatura. Sobre la antigüedad laboral, el 42.6\%, llevan trabajando entre 7-12 años. El 67.8\% laboran en el turno matutino, con categoría las enfermeras generales representan el 78.9\%, información que se describe en la Tabla 2.

En relación con el tiempo estimado de dedicación por cada enfermera en las intervenciones durante la jornada laboral, se identificaron diferencias en la atención según los grados de la escala de TISS-28. En el Grado II de complejidad corresponde el 13.7\% de los pacientes del género femenino, $20.7 \%$ del grupo de edad 36 a 65 años, el 13.3\% pertenecen al servicio de cirugía general y $11.5 \%$ al servicio de medicina interna. El Grado III corresponde a los pacientes con estancia hospitalaria de una 
Tabla 1. Características sociodemográficas de la población atendida

\begin{tabular}{|c|c|c|c|}
\hline Variables & & Frecuencia & $\%$ \\
\hline \multirow[t]{2}{*}{ Género } & Masculino & 124 & 45.9 \\
\hline & Femenino & 146 & 54.1 \\
\hline \multirow[t]{7}{*}{ Edad (años) } & Infantil (< 1 año) & 12 & 4.4 \\
\hline & Preescolar (1 - 4 años) & 4 & 1.5 \\
\hline & Niñez (5 - 13 años) & 2 & 0.7 \\
\hline & Adolescente (14 - 17 años) & 1 & 0.4 \\
\hline & Adulto Joven (18 - 35 años) & 32 & 11.8 \\
\hline & Adulto (36 - 64 años) & 136 & 50.4 \\
\hline & Adulto Mayor (> 65 años) & 83 & 30.7 \\
\hline \multirow[t]{2}{*}{ Servicio } & Medicina Interna & 162 & 60.0 \\
\hline & Cirugía General & 108 & 40.0 \\
\hline \multirow[t]{5}{*}{ Día Estancia } & 1 semana & 206 & 76.3 \\
\hline & 2 semanas & 44 & 16.3 \\
\hline & 3 semanas & 10 & 3.7 \\
\hline & 4 semanas & 6 & 2.2 \\
\hline & 5 y más semanas & 4 & 1.5 \\
\hline
\end{tabular}

semana, el servicio de medicina interna registro mayor demanda de tiempo en atención de personal de enfermería con $55.2 \%$, pertenecen a este grado el 38.1\% de pacientes del género femenino y $37.4 \%$ de los adultos, información que se encuentra descrita en la Tabla 3.

\section{DISCUSIÓN}

El uso de escalas para medir la carga de trabajo enfermero se ha utilizado para determinar el tiempo y la relación paciente-enfermera. De acuerdo con la población estudiada los pacientes adultos, así como adultos mayores fueron los que más atenciones de enfermería y servicios de hospital requirieron.

A diferencia de lo referido por la literatura internacional los servicios de cuidados intensivos o quirúrgicos son los más demandantes de la atención de enfermería ${ }^{1,5-8,14-16}$. En nuestro estudio, el servicio de medicina interna y los pacientes con una sobre estancia hospitalaria de 3 semanas son las variables con mayor demanda de intervenciones realizadas por enfermería. Condición que se justifica pues son pacientes con alteraciones fisiopatológicas que han alcanzado un nivel de severidad que puede convertirse en una amenaza actual o potencial para su vida o rehabilitación. Además, requieren de cuidados médicos y de enfermería constante, ya que consumen una elevada cantidad de recursos tanto materiales como humanos.

Por otro lado, algunos estudios coinciden al evaluar las intervenciones terapéuticas y utilizar la connotación carga de trabajo en los profesionales de enfermería, de manera que esta se encuentra directamente relacionada con la recuperación del paciente ${ }^{17,18}$. Se han desarrollado modelos con el fin de predecir las complejidades de las actividades de enfermería para establecer la relación paciente-enfermera en la UCI, evaluar el uso común de las camas y conocer de manera oportuna las necesidades futuras en el servicio ${ }^{19,20}$. Asimismo, el principal objetivo de la escala TISS-28 es valorar la gravedad de los pacientes críticos, permitiendo reducir las cifras de morbilidad y mortalidad ${ }^{21-23}$. 
Tabla 2. Caracteristicas sociodemográficas del profesional de enfermería

\begin{tabular}{|c|c|c|c|}
\hline Variables & & Frecuencia & $\%$ \\
\hline \multirow[t]{2}{*}{ Género } & Femenino & 199 & 73.7 \\
\hline & Masculino & 71 & 26.3 \\
\hline \multirow[t]{4}{*}{ Edad (años) } & 18 a 29 & 50 & 18.5 \\
\hline & 30 a 39 & 143 & 53.0 \\
\hline & 40 a 49 & 69 & 25.5 \\
\hline & 50 y más & 8 & 3.0 \\
\hline \multirow[t]{3}{*}{ Escolaridad } & Secundaria & 7 & 2.6 \\
\hline & Preparatoria & 144 & 53.3 \\
\hline & Licenciatura & 119 & 44.1 \\
\hline \multirow[t]{5}{*}{ Antigüedad laboral } & 1 a 6 años & 78 & 28.9 \\
\hline & 7 a 12 años & 115 & 42.6 \\
\hline & 13 a 18 años & 70 & 25.9 \\
\hline & 19 a 24 años & 3 & 1.1 \\
\hline & 25 o más años & 4 & 1.5 \\
\hline \multirow[t]{2}{*}{ Turno laboral } & Matutino & 183 & 67.8 \\
\hline & Vespertino & 87 & 32.2 \\
\hline \multirow[t]{2}{*}{ Estado Civil } & Soltero & 110 & 40.7 \\
\hline & Casado & 160 & 59.3 \\
\hline \multirow[t]{4}{*}{ Categoría laboral } & Enfermera General & 213 & 78.9 \\
\hline & Enfermera Especialista Intensivista & 41 & 15.2 \\
\hline & Enfermera Especialista Pediátrica & 11 & 4.0 \\
\hline & Enfermera Especialista Quirúrgica & 5 & 1.9 \\
\hline
\end{tabular}

Con base en lo anterior, identificamos que el TISS-28 es una herramienta válida para evaluar al resto de los servicios hospitalarios, pues como predictor de la carga laboral valora las condiciones de cada servicio por parte del equipo de enfermería.

De acuerdo con los datos obtenidos en este estudio, los grados II y IV en el servicio de pediatría fueron los que representaron mayor carga de trabajo. Estos hallazgos son similares con otros autores, quienes estiman cargas de trabajo de enfermería de 11 horas en pacientes pediátricos. De igual forma, haciendo uso del TISS-28 mencionan lo imprescindible que es el personal de enfermería para satisfacer las necesidades en el área pediátrica, además de que sirven como predictores de mortalidad, de modo que es importante la vigilancia estrecha de dichos profesionales en este servicio ${ }^{11,21-25}$.

El grado III del TISS-28 es el que más tiempo requiere de atención del profesional de enfermería, por lo que se puede inferir que a mayor grado, mayor gravedad y mayores cuidados enfermeros. En estudios recientes en un turno de ocho horas la atención fue con un tiempo mínimo de 3.71 horas y máximo de 7.95 horas, con promedio de 5.9 horas, en pacientes con grado III ${ }^{15}$. La complejidad de las fisiopatologías que se atienden en los servicios de medicina interna alcanzan niveles de gravedad 
Tabla 3. Grados de complejidad de las intervenciones

en el cuidado de enfermeria según los grados del TISS-28

\begin{tabular}{|c|c|c|c|c|c|c|c|}
\hline \multirow[t]{2}{*}{ Variables } & & \multicolumn{2}{|c|}{ Grado II } & \multicolumn{2}{|c|}{ Grado III } & \multicolumn{2}{|c|}{ Grado IV } \\
\hline & & $\mathrm{Fe}$ & $\%$ & $\mathrm{Fe}$ & $\%$ & $\mathrm{Fe}$ & $\%$ \\
\hline \multirow[t]{2}{*}{ Género } & Masculino & 30 & 11.1 & 89 & 32.9 & 5 & 1.8 \\
\hline & Femenino & 37 & 13.7 & 103 & 38.1 & 6 & 2.2 \\
\hline \multirow{7}{*}{$\begin{array}{l}\text { Grupo de } \\
\text { edad }\end{array}$} & Infantil (0-11 meses) & 0 & 0 & 12 & 4.4 & 0 & 0 \\
\hline & Preescolar (1-4 años) & 3 & 0 & 1 & 0.4 & 0 & 0 \\
\hline & Niñez (5-13 años) & 0 & 0 & 1 & 0.4 & 1 & 0.4 \\
\hline & Adolescente (14-17 años) & 0 & 0 & 1 & 0.4 & 0 & 0 \\
\hline & Adulto Joven (18-35 años) & 8 & 2.9 & 19 & 7.0 & 5 & 1.8 \\
\hline & Adulto (36-64 años) & 31 & 11.5 & 101 & 37.4 & 4 & 1.4 \\
\hline & Adulto Mayor (> 65 años) & 25 & 9.2 & 57 & 21.1 & 1 & 0.4 \\
\hline \multirow[t]{2}{*}{ Servicio } & Servicio Medicina Interna & 31 & 11.5 & 125 & 46.3 & 6 & 2.2 \\
\hline & Servicio Cirugía General & 36 & 13.3 & 67 & 24.8 & 5 & 1.8 \\
\hline \multirow{5}{*}{$\begin{array}{l}\text { Tiempo de } \\
\text { estancia }\end{array}$} & 1 semana & 49 & 18.2 & 149 & 55.2 & 8 & 2.9 \\
\hline & 2 semanas & 10 & 3.7 & 31 & 11.5 & 3 & 1.1 \\
\hline & 3 semanas & 5 & 1.9 & 5 & 1.8 & 0 & 0 \\
\hline & 4 semanas & 1 & 0.4 & 5 & 1.8 & 0 & 0 \\
\hline & 5 y más semanas & 2 & 0.7 & 2 & 0.7 & 0 & 0 \\
\hline
\end{tabular}

que pueden transformarse en una amenaza potencial para la vida de estos pacientes, lo que implica la presencia de riesgos relacionados con la carga laboral ${ }^{12,26,27}$.

A su vez, es importante resaltar que la clasificación encontrada en los pacientes del estudio reflejó diferencias consideradas entre los diferentes servicios estudiados, lo que refleja que, aunque no son servicio exclusivo para atender pacientes críticos como el de Cuidados intensivos, el servicio de medicina interna atiende pacientes con clasificación III y IV, evidenciando que el paciente requiere diversas intervenciones. En consecuencia, se destina más tiempo de cuidado enfermero, dando con ello una carga mayor de trabajo para el personal de enfermería, cuyos indicadores de distribución del personal no son adecuados, por lo que es necesario emplear el TISS-28 como herramienta de evaluación.

\section{CONCLUSIONES}

El instrumento TISS-28 sintetiza la suma de las intervenciones que realiza el profesional de enfermería y cuantifica la gravedad del paciente, dando como resultado el tiempo que enfermería requiere dedicar para su atención, lo que permite reflexionar sobre el tiempo que se dedica a un paciente según su gravedad, el cuidado de enfermería y la realidad hospitalaria.

En ese sentido, es importante cuestionarse y encontrar soluciones a las siguientes preguntas: 1) ¿la distribución del profesional de enfermería por servicio hospitalario es la idónea?, 2) ¿es congruente la distribución del personal de enfermería con las necesidades de cuidado de los pacientes por cada turno?, 3) ¿el hospital cuenta con instrumentos y/o escalas de evaluación como el TISS-28 para la distribución de la plantilla de enfermería? 
Con base en lo anterior, podemos concluir que existen diferentes factores como la relación enfermera-paciente de la unidad de estudio y la enfermera profesional y no profesional en áreas críticas, lo que influye en que se presente la carga de trabajo vinculada en forma estrecha con el grado de gravedad según el TISS-28. En este estudio se apreció que, a menor edad del paciente, mayor intervención requirió del personal de enfermería.

Finalmente, el TISS-28 es una herramienta indispensable para determinar el grado de complejidad en las intervenciones que se realizan en pacientes en estado crítico y el tiempo de atención dependiendo de la gravedad, por lo que permite conocer el número correcto asignado de enfermería-paciente por cada turno de trabajo.

\section{LIMITACIONES DEL ESTUDIO}

Una de las limitaciones en las intervenciones realizadas por el profesional de enfermería fue no encontrar registrada las indicaciones médicas, lo que puede convertirse en una posible interferencia en la ponderación del instrumento.

\section{RESPONSABILIDADES ÉTICAS}

Protección de personas o animales. Los autores declaran que en esta investigación no se realizó ningún experimento con seres humanos.

Confidencialidad. Los autores declaran que en este documento no aparecen los datos de los participantes, garantizando su anonimato y el manejo confidencial de la información.

Conflicto de intereses. Los autores declaran no tener conflicto de intereses.

Financiamiento. Ninguno.

\section{REFERENCIAS}

1. Pérez-Niculcar PN, Zuazua-Rico D. Carga de trabajo de enfermería en la unidad de cuidados intensivos de adultos. Revisión sistemática. Nure Inv. 2018;15(93): 1-17.

2. Pérez-Gutiérrez N. Análisis de mortalidad de pacientes en unidad de cuidados intensivos en un hospital del departamento del Meta, Colombia. Investigaciones andina. 2015; 18(33): 1605-24. https://doi.org/10.33132/01248146.644

3. Lagos-Garrido ME, Paravic-Klijn T. Generación, difusión y transferencia del conocimiento de enfermería a la práctica del cuidado. Cienc. enferm. 2015; 21(2): 127-34.

http://dx.doi.org/10.4067/S0717-95532015000200012

4. Rodríguez-Jiménez S, Cárdenas-Jiménez M, Pacheco-Arce AL, Ramírez-Pérez M. Una mirada fenomenológica del cuidado de enfermería. Enferm. univ. 2014; 11(4): 145-53.

https://doi.org/10.1016/S1665-7063(14)70927-3

5. Pujante-Palazón I, Rodríguez-Mondéjar JJ, Armero-Barranco D, Sáez-Paredes P. Prevención de neumonía asociada a ventilación mecánica, comparación de conocimientos entre tres unidades de críticos. Enferm. Intensiva. 2016; 27(3): 120-8. https://doi.org/10.1016/j.enfi.2015.10.001

6. Oliveira Moscheta-Campagner A, Celiny Ramos-Garcia P, Pedro-Piva J. Aplicação de escores para estimar carga de trabalho de enfermagem em unidade de terapia intensiva pediátrica. Rev Bras Ter Intensiva. 2014; 26(1): 36-43. https://doi.org/10.5935/0103-507X.20140006

7. Molina-Mula J, Vaca-Auz J, Muñoz-Navarro P, Cabascango-Cabascango K, Cabascango-Cabascango C. Gestión de la calidad de los cuidados de enfermería hospitalaria basada en la evidencia científica. Index Enferm. 2016; 25(3): 151-5. https://bit.ly/33yZIgN 
8. Reyes-Segura SM, Parellada-Blanco J, García-Vega ME, Martínez-Verzonis N. Aplicación del sistema pronóstico TISS-28 en salas de atención al grave. Rev Cub Med Int Emerg. 2015; 1(4): 53-66.

https://bit.ly/2OxMZif

9. Nieri AS, Manousaki K, Kalafati M, Grilio-Padilha K, Stafseth SK, Katsoulas T, et al. Validation of the nursing workload scoring systems "Nursing Activities Score" (NAS), and "Therapeutic Intervention Scoring System for Critically Ill Children" (TISS-C) in a Greek Paediatric Intensive Care Unit. Intensive Crit Care Nurs. 2018; 48: 3-9.https://doi.org/10.1016/j.iccn.2018.03.005

10. Cullen DJ, Civetta JM, Briggs BA, Ferrara LC. Therapeutic intervention scoring system: a method for quantitative comparison of patient care. Crit Care Med. 1974; 2(2): 57-60.

11. Vivanco-Allende A,Rey C, Concha A,Martínez-Camblor P, Medina A,Mayordomo-Colunga J.Validación de la escala simplificada de puntuación de intervenciones terapéuticas (TISS-28) en niños críticamente enfermos. An pediatr. 2020: 92(6): 339- 44. https://doi.org/10.1016/j.anpedi.2019.10.002

12. Laus AM, Gonçalves-Menegueti M, Martins MA, Dias Pedreschi-Chaves L, Camelo SH. Fatores associados à carga de trabalho de enfermagem em terapia intensiva: quais se devem considerar? Rev enferm UFPE on line. 2017; 11(Supl. 12): 5305-11.

https://doi.org/10.5205/1981-8963-v11112a22847p5305-5311-2017

13. Tekdos-Seker Y, Hergunsel O, Bostanci I, Zeydan A. Utility of the therapeutic intervention scoring system-28 to predict mortality in intensive care units. EJMO. 2018; 2(1): 35-9.

http://doi.org/10.14744/ejmo.2017.00719

14. Royo-Puerto M, Araiz-Marín AB, Palacios-Laseca M, Gago-Gallego N, Latorre-Navascuez R, BascuasHernández J. Análisis de la carga de trabajo y cálculo de la ratio enfermera-paciente en cuidados intensivos en pacientes tratados con hipotermia tras parada cardiaca extrahospitalaria. Enferm Cardiol. 2019; 26(76): 43-53.

15. Saltos-Rivas M, Pérez-Cardoso CN, Suárez-Mella R, Linares-Giler SM. Análisis de la carga laboral del personal de enfermería, según gravedad del paciente. Rev. cuba. enferm. 2018; 34(2): 43-53. https://bit.ly/36xyMdo

16. Martín-Sánchez FJ, Carbajosa V, Llorens P, Herrero P, Jacob J, Miró Ò, et al. Tiempo de estancia prolongado en los pacientes ingresados por insuficiencia cardiaca aguda. Gac. sanit. 2016; 30(3): 191-200. http://dx.doi.org/10.1016/j.gaceta.2016.01.003

17. Justo-Martínez M, Domínguez-Orozco V, Pérez Castro-y Vásquez JA, Gusman-Morales E. Eficacia de las intervenciones de enfermería dependientes en el paciente de terapia intensiva. Rev. Conamed. 2018; 3(16): 3-7. https://bit.ly/37xwk64

18. Fátima-Perão O, Camargo-Bub MB, Hernández-Rodríguez A, Zandonadi GC. Gravidade de pacientes e carga de trabalho de enfermagem em unidade de terapia intensiva. Cogitare Enferm. 2014; 19(2): 261-8. https://bit.ly/3fZEFn7

19. Romero-Massa E, Lorduy-Bolivar JP, Pájaro-Melgar C, Pérez-Duque CA. Relación entre la carga laboral de enfermería y la gravedad del paciente en unidades de cuidado intensivo de adultos. Aquichan. 2011; 11(2): 173-86. https://bit.ly/2I3AsC9

20. Tavares-Araujo M, Baia-Henriques AV, Cancio-Velloso IS, Ferreira-de Queiroz C, França-Nonato L. Carga de trabalho e custo de uma equipe de enfermagem em terapia intensiva. Arq. Ciênc. Saúde. 2016; 23(4): 21-6. https://doi.org/10.17696/2318-3691.23.4.2016.385

21. Stochero-Velozo KD, Celiny Ramos-Garcia P, Pedro-Piva J, Holmer-Fiori H, Drescher-Cabral D, Einloft PR, et al. Scores TISS-28 versus NEMS to size the nursing team in a pediatric intensive care unit. Einstein (São Paulo). 2017; 15(4): 470-5. http://doi.org/10.1590/S1679-45082017AO4O28 
22. Chacón-Lozsán FJ, Delgado C, Castillo Y, Pacheco PA, Sánchez-Narváez MC. Score Therapeutic Intervention Scoring System de ingreso a la unidad de cuidados intensivos como predictor de mortalidad intra-hospitalaria. Acta colombiana de cuidado intensivo. 2015; 15(4): 307-11.

https://doi.org/10.1016/j.acci.2015.07.001

23. Valls-Matarín J, Salamero-Amorós M, Roldán-Gil C. Análisis de la carga de trabajo y uso de los recursos enfermeros en una unidad de cuidados intensivos. Enferm. Intensiva. 2015; 26(2): 72-81. https://doi.org/10.1016/j.enfi.2015.02.002

24. Cabral-Ferreira P, Machado RC, Fortes-Vitor A, Brendão de Cavalho-Lira AL, Soares-Martins QS. Nursing measure in intensive care unit: evidence about the nursing activites score. Rev Rene. 2014; 15(15): 888-97. http://dx.doi.org/10.15253/2175-6783.2014000500019

25. Reynaldos-Grandon KL, Toffoletto MC, Molina-Muñoz Y, Grillo-Padilha K, Sánchez-Aguiera NA, Maldonado-Mardones EI. Adaptación transcultural para Chile del nursing activities score para medir la carga de trabajo de enfermería en unidades de cuidados intensivos. Cienc. enferm. 2018; 24: 1-14. http://doi.org/10.4067/so717- 95532018000100212

26. Cuadros-Carlesi K, Grillo-Padilha K, Toffoletto MC, Henriquez-Roldan C, Canales-Juan MA. Ocurrencia de incidentes de seguridad del paciente y carga de trabajo de enfermería. Rev. Latino-Am. Enfermagem. 2017; 25(6): 1-8. https://doi.org/10.1590/1518-8345.1280.2841

27. Wang ZW, Zheng J, You LM, Wang YX, Gao MR, Guan XD. Evaluation of the simplified therapeutic intervention scoring system: Chinese version. Intensive Crit Care Nurs. 2018; 45: 85-90.

https://doi.org/10.1016/j.iccn.2017.09.009 